matching on civil registration number and date 97 patients were found in all three registries, 140 only in hospitals trauma, 8683 only in pre-hospital and 8147 only in ambulance data. For $69 \%$ of the trauma registry patients location of occurrence where known.

Conclusions For the present geographical region in Denmark pooling data from these registries seems a promising way for identifying place of occurrence. However, immediate merge were only valid with manual cumbersome review. Further analysis must look into whether the recorded locations are valid indications of actual places and gives sufficient input to the planning of preventive measures in municipalities.

\section{NEW EMERGING PERSPECTIVES ON RAOD SAFTY MANAGMENT: WHAT LIGHT CAN COMPLEXITY SCIENCE TROW?}

Nastaran Keshavarz Moahmmadi. Shaid Behesti University of Medical Sciences, Iran

\subsection{6/injuryprev-2016-042156.532}

Background The words "complexity" and" complex system are increasingly used in road safety and transport system literature. They refer to the acknowledgement of the complexity of the multi-causal nature of road safety issues, and their solutions. The terms have also been used in describing the complexity of changing individual behaviours or policies, anticipating and assessing the consequences of interventions. Hence, it seems rational turning to complexity science for insights.

Description of the problem Complexity science is about complex adaptive systems (CAS). It defines a complex adaptive system as a collection of autonomous, interactive and adaptive agents that act freely in diverse ways, and are unpredictable and, non-linear but interconnected. It is considered an ever changing system, typically with fuzzy boundaries which is built of multilayer nested systems.

Changes Utilising the lenses of complexity, a road traffic system is perceived as a dynamic complex adaptive system formed of many diverse interdependent agents including individuals, culture, environment, policies, vehicles and roads which constantly change. In addition, any change in one aspect or component of this system can significantly change other parts and so the whole system. This new understandings provides new explanations for current road safety issues leading us to new understanding of potential adaptable solutions.

Conclusions This paper will explain this new understanding. By doing this, it suggests that utilising concepts of complexity science is a promising line of inquiry to explore successful approaches to context and time dependent road safety interventions. It also argues that it is not possible to remove the complexity, but we need to harness it in order to be able to develop more safe transport systems across all countries and communities.

\section{FRACTURE PATTERNS IN YOUNG CHILDREN AFTER MOTOR VEHICLE CRASHES}

Michael Wilson, Winesh Ramphal, Monique van Dijk, Alp Numanoglu, Arjan B van As. Trauma Unit, Red Cross War Memorial Children's Hospital, University of Cape Town

\subsection{6/injuryprev-2016-042156.533}

Background In Africa, road traffic injuries rank second to interpersonal violence and the road traffic fatality rate is higher than for any WHO region. The aim of this study is to explore potential differences in number and patterns of fractures between childhood survivors and non-survivors after a motor vehicle crash in view of developing targeted and more effective prevention strategies.

Methods Total body radiographs were obtained of all children under 13 years presenting to our trauma unit after a motor vehicle crash during a 30 month period between January 2010 and July 2012. In addition, total body radiographs were obtained from the mortuary of all children under the age of 13 who demised after a motor vehicle crash. The number and types of fractures as well as any other skeletal malformations were compared and evaluated. Approval was obtained from our University Ethics Committee.

Results A total of 1045 children were assessed; 1007 presenting to the trauma unit, and 38 directly to the mortuary. Twenty patients presenting to the trauma unit did not survive. The total amount of non survivors was 58 but 6 were excluded since radiographs were not available. From the 987 survivors 79 were excluded because the radiographs were unavailable. The average age of children was 6.2 years (SD 3.1; Range 0-12). Fractures were far more common in non-survivors than survivors $(78.8 \%$ vs $46.7 \%)$. Fractures of the Skull ( $p<0.0001)$, Cervical Spine ( $\mathrm{p}<0.0001)$, Thoracic Vertebrae $(\mathrm{p}<0.0001)$, Trunk including Shoulder ( $\mathrm{p}<0.0001)$, Lower Arm $(\mathrm{p}<0.002)$, Upper Arm $(p<0.3)$ were statistically significantly more frequent in non-survivors than in survivors. Fractures of Facial bones, Elbow, Wrist, Hand, Pelvis, Femur, Knee, Foot and Ankle were different but not statistically significant in survivors and non-survivors.

Conclusions This is the first study documenting and comparing fracture patterns with outcome (survivors and non-survivors) in young children after motor vehicle crash. Since motor vehicle crashes are the most common cause of unnatural childhood death it is important to study fracture patterns in order to understand the injury mechanism and develop preventative strategies. This study indicates that skull, cervical spine and torso fractures are more intricately associated with severe morbidity and mortality in children than fractures of other body regions. The upper torso, head and neck are the body regions most vulnerable in young children and requiring most protection.

\section{RESULTS OF THE "DECADE OF ROAD SAFETY ACTION" IN COLOMBIA. 2010-2015}

${ }^{1}$ Gustavo Cabrera, ${ }^{2}$ Jorge Rodríguez, ${ }^{2}$ Fredy Camelo. ${ }^{1}$ University of Antioquia, Colombia; ${ }^{2}$ Pontifical Xaverian University, Colombia

\subsection{6/injuryprev-2016-042156.534}

Background In the 1990's, the United Nations set the standards for confronting the increasing issue of traffic injuries and fatalities (TIF). In Colombia, the 2013-2021 National Road Safety Plan was created to implement guidelines and facilitate inter-sector coordination of the strategic pillars proposed in the program Decade of Road Safety Action-DRSA. This study shows the results of the first five years of the DRSA program in Colombia. Methods This descriptive, longitudinal study analyses the behaviour of TIF in Colombia from 2010 to 2015. The injury and fatality records were obtained from the Colombian National Institute of Legal Medicine and from the Colombian National Statistics Department. The number of vehicles was obtained from the Unified National Transit Registry. Adjusted fatality rates were estimated. A lineal regression model was made to correlate the 